\title{
The epidemiology of porcine Taenia solium cysticercosis in communities of the Central Highlands in Vietnam
}

Dinh Ng-Nguyen ${ }^{1,2^{*}}$, John Noh ${ }^{3}$, Kathleen Breen ${ }^{4}$, Mark Anthony Stevenson $^{1}$, Sukwan Handali ${ }^{3}$ and Rebecca Justine Traub ${ }^{1}$

\begin{abstract}
Background: Taenia solium cysticercosis, recognized as a neglected tropical disease by the WHO, is distributed mostly in developing countries of Latin America, sub-Saharan Africa and Asia. Pigs and humans act as intermediate hosts, acquiring T. solium cysticerci (larval stage) in their tissue, through the ingestion of T. solium eggs shed in the faeces of humans infected with adult tapeworms. The disease has a negative impact on rural economies due to losses in productivity arising from human disease, pork carcass condemnations and loss of market access. The aim of this study was to estimate the prevalence of T. solium cysticercosis in pigs in Dak Lak Province in the Central Highlands of Vietnam and to identify household level characteristics associated with T. solium porcine cysticercosis.

Methods: This was a cross-sectional study of household pigs in three districts of Dak Lak Province. A total of 408 households in six villages in three districts were visited between June and October 2015. A questionnaire was administered to the head of each household, and within each household, serum samples were collected from three pigs. Serum samples were analyzed using the recombinant T24H antigen in enzyme-linked immunoelectrotransfer blot assay and lentil lectin purified glycoprotein in EITB assay. A Bayesian, mixed-effects logistic regression model was developed to identify management factors associated with the probability of a household having at least one cysticercosis-positive pig.
\end{abstract}

Results: The prevalence of porcine T. solium cysticercosis in this study was low at 0.94 [95\% confidence interval (Cl) 0.51-1.68] cases per 100 pigs at risk, in agreement with other studies conducted throughout Vietnam. Scavenging of food and coprophagy were associated with T. solium cysticercosis [odds ratios 1.98 (95\% Crl: 0.55-4.74) and 2.57 (95\% Crl: 1.22-4.66), respectively].

Conclusions: This study proves that the seroprevalence of porcine cysticercosis in Dak Lak Province was as low as that of other studies conducted throughout Vietnam. Scavenging of food and coprophagy are modifiable factors, providing the opportunity to decrease the prevalence of porcine cysticercosis further in the province.

Keywords: Taenia solium, Porcine cysticercosis, Epidemiology, Vietnam

\footnotetext{
*Correspondence: ngocn4@student.unimelb.edu.au; theeveret@gmail.com

${ }^{1}$ Faculty of Veterinary and Agricultural Sciences, University of Melbourne,

Parkville, Victoria 3052, Australia

${ }^{2}$ Faculty of Animal Sciences and Veterinary Medicine, Tay Nguyen University,

Buon Ma Thuot, Dak Lak, Vietnam

Full list of author information is available at the end of the article
}

(c) The Author(s). 2018 Open Access This article is distributed under the terms of the Creative Commons Attribution 4.0 International License (http://creativecommons.org/licenses/by/4.0/), which permits unrestricted use, distribution, and reproduction in any medium, provided you give appropriate credit to the original author(s) and the source, provide a link to the Creative Commons license, and indicate if changes were made. The Creative Commons Public Domain Dedication waiver (http://creativecommons.org/publicdomain/zero/1.0/) applies to the data made available in this article, unless otherwise stated. 


\section{Background}

Taenia solium cysticercosis is recognized as a neglected tropical disease by the WHO [1]. It is distributed mostly in developing countries of Latin America, sub-Saharan Africa and Asia [2]. Pigs and humans act as intermediate hosts, acquiring $T$. solium cysticerci (larval stage) in their tissue, through the ingestion of $T$. solium eggs shed in the faeces of humans infected with adult tapeworms. Consumption of raw and/or undercooked pork with active $T$. solium cysticerci may result in $T$. solium taeniasis in humans. The presence of porcine cysticercosis impacts negatively on an economy due to costs arising from carcass condemnation and negative impacts on market access and trade of pork.

Although Vietnam is located in a region endemic for T. solium [3], the data on porcine cysticercosis are limited. In addition, it is not clear whether porcine cysticercosis is endemic in the country. During 1994 to 2005, the highest reported prevalence of porcine cysticercosis in Vietnam among four carcass-based studies was less than 1\% [4]. These estimates were, for the most part, based on studies conducted in commercial slaughterhouses, and therefore do not reflect the prevalence of cysticercosis in pig populations in rural communities that are not processed in commercial slaughterhouses [5]. Of the relatively small number of studies that have quantified the prevalence of porcine cysticercosis in the country, most were conducted prior to 2003 with a focus on the north of Vietnam [4]. To the best of our knowledge, the only study of porcine cysticercosis in the south of Vietnam was carried out in 1994 [6].

Dak Lak Province is located in the Central Highlands in the south of Vietnam. A recent study in the communities of the province demonstrated an apparent prevalence of $1.2 \%$ T. solium taeniasis [7]. In most communities in the province, pigs are free-roaming and outdoor defaecation is common [8]. These characteristics are conducive for infection and transmission of T. solium cysticercosis to pigs. The aim of this study was to estimate the prevalence of cysticercosis in pigs in Dak Lak and to identify household level characteristics associated with $T$. solium porcine cysticercosis.

\section{Methods}

\section{Study site and sampling}

Fieldwork was conducted between June and October 2015 in Krong Nang, M'Drak and Buon Don districts in Dak Lak Province, Vietnam. These districts were chosen as the study sites based on their diverse geographical characteristics. The study sites have been described in detail elsewhere [7]. In brief, M'Drak is located in the east of Dak Lak Province with an average altitude of 400 $\mathrm{m}$ to $500 \mathrm{~m}$ and has a tropical monsoon climate typical of the Vietnam's Central Coast. Krong Nang is situated in the north of the province at an altitude of $800 \mathrm{~m}$. Buon Don, situated to the west of the province with an average elevation of $330 \mathrm{~m}$ and has a hot and dry climate. The standard of living in this area of Vietnam is generally poor. Open defaecation using outdoor pit latrines is common practice and livestock access to these latrine areas is usually unrestricted. The practice of non-confinement of pigs and cattle is common with slaughter activities often carried out in backyards [4].

A sampling frame listing the name of all villages in the three study districts was obtained from Sub-Department of Animal Health office. Villages eligible for sampling comprised those with more than 1000 pigs, as recorded by the Sub-Department of Animal Health within the Ministry of Agriculture. All eligible villages within each of the three study districts were assigned a number and two numbers were chosen at random to select villages from each district for inclusion in the study. Within each selected village, a list of householder names was obtained from the respective village head person, and each householder name was then coded with a number (the number of households per village ranged from 200 to 300). A sheet of paper was drawn up into squares and each square numbered from 1 to 300 . The squares were cut into pieces and placed face-down on a table. The village head was then asked to select between 100 and 140 squares. The number on each selected square identified each household to be sampled. All selected households were visited several days before sampling to obtain consent from participants. Within each district, blood samples were collected from pigs in each of the study households. At the time of each household visit pig owners were asked to complete a questionnaire on the number and type of pigs kept and details of demography, husbandry practices, and diet (Table 1). All questionnaires were conducted in local Vietnamese phraseology and their validity pre-tested on 30 pig owners in another community in Dak Lak Province before application to the field survey. In addition, interviewers were trained before administering the questionnaires. Pigs were selected at random by the member of research group for blood sampling. Pigs that were pregnant or ill, and pigs aged less than 2 months of age were excluded from sampling. At the time of each household visit $10 \mathrm{ml}$ of blood was obtained from the cranial vena cava of each pig into plain blood collection tubes. The blood samples were allowed to clot at ambient temperature prior to centrifugation at $3200 \times g$ for $5 \mathrm{~min}$ to separate serum. Serum was dispensed into $1.5 \mathrm{ml}$ aliquots and stored at $-20{ }^{\circ} \mathrm{C}$ until use.

\section{Sample size estimation}

The aim of this study was to estimate the prevalence of porcine T. solium cysticercosis in Dak Lak Province. 
Table 1 Details of information about general and pig information

\begin{tabular}{ll}
\hline Requesting information & Detail \\
\hline General information & $\begin{array}{l}\text { Address of house; number of people per household; presence and type and location of toilet; number } \\
\text { of livestock per household; vegetables and crops management (source of water for irrigation, kind of } \\
\text { manure for vegetables and crops); coordinate of household, pigsty, toilet and vegetable patch; elevation } \\
\text { of household; number of dogs; location of defecation for dogs; efforts to disinfect dog's faeces; } \\
\text { observation of Taenia proglottids in dog's faeces }\end{array}$ \\
$\begin{array}{ll}\text { Pig information } \\
\text { Demography } \\
\text { Husbandry management }\end{array}$ & $\begin{array}{l}\text { Sex; age; breed; number of pigs } \\
\text { The presence and location of pigsty; whether pig confined or free-roaming; roaming range of pigs; } \\
\text { whether pigs eat human faeces; whether pigs have access to human defecation area }\end{array}$ \\
Diet management & $\begin{array}{l}\text { Drinking water sources for pigs, kind of food for pigs (commercial or handmade bran, scavenging), } \\
\text { feeding raw vegetables to pigs or not, vegetables washed before feeding pigs }\end{array}$ \\
\hline
\end{tabular}

Based on a previous slaughterhouse based survey by Van De at al. [9], the prevalence of porcine cysticercosis was assumed to be $10 \%$. Assuming 95\% certainty that this estimate was within $5 \%$ of the actual population prevalence (i.e. cysticercosis prevalence ranged from $5 \%$ to $15 \%)$ and ignoring the possibility that cysticercosis positive pigs were clustered within households, we estimated that a total of 384 pigs were required to be sampled. We then assumed the average number of pigs eligible for random sampling per household was at least three and an intra-class correlation coefficient for $T$. solium cysticercosis of 0.07 [10] returning a design effect of 1.14 . Our revised sample size, accounting for the likelihood that porcine cysticercosis clusters within households, was $384 \times 1.14=438$ for each of the three study districts.

\section{Laboratory procedures}

Pig serum samples were analyzed using the recombinant $\mathrm{T} 24 \mathrm{H}$ antigen in enzyme-linked immunoelectrotransfer blot (rT24H-EITB) assay as described by Noh et al. [11] and lentil lectin purified glycoproteins in EITB (LLGP-EITB) assay as previously described by Tsang et al. [12] and Gonzalez et al. [13]. Both the LLGP-EITB and rT24H-EITB assay are immunoblot methods. The LLGP-EITB detects antibodies to one or more of seven lentil lectin purified glycoproteins (LLGPs), namely GP50, GP42, GP24, GP21, GP18, GP14 and GP13 which are present in the soluble fraction of an extract of $T$. solium cysticerci [11]. Reaction to any of these 7 glycoprotein antigens is considered positive. The rT24H-EITB assay detects antibodies against $\mathrm{rT} 24 \mathrm{H}$ antigen derived from 24- and 42-kDa glycoproteins of the LLGPs [14].

To ascertain the analytical specificity of the rT24H antigen, we subjected 29 cysticercosis-negative USA pig sera, 12 necropsy-positive $T$. solium-positive Peruvian pig sera and $4 T$. hydatigena necropsy-positive Vietnamese pig sera to the rT24H-EITB. All USA pig sera and Vietnamese $T$. hydatigena pig sera were negative for the rT24H-EITB, and all Peruvian T. solium positive pig sera were positive on the rT24H-EITB. These preliminary results provided the basis of results show that under experimental conditions, the rT24H-EITB do not cross-react to pig sera with $T$. hydatigena.

Individual serum samples were screened in pools of four using the rT24H antigen in EITB assay format to detect the presence of antibodies against $T$. solium cysticerci. The rT24H antigen was utilized in the EITB assay as it offers the best overall diagnostic performance compared with other recombinant or synthetic antigens based on our preliminary data and previous human-based studies $[15,16]$. Individual serum sample from each positive pools were then re-screened using rT24H-EITB and LLGP (native antigen of $T$. solium cysticerci) antigens. The LLGP-EITB has been used as the reference standard assay for serological diagnosis of $T$. solium cysticercosis in humans and pigs that has the specificity of $100 \%$ and sensitivity between $98-100 \%[12,13]$. Jayashi et al. [17] when validating the LLGP-EITB for naturally acquired porcine cysticercosis pointed out that the LLGP-EITB achieves optimal sensitivity of 78\% (95\% CI: $52-94 \%)$ and specificity of $76 \%$ (95\% CI: 66-85\%) when the assay reacts to $\geq 3$ of 7 LLGP antigens. The diagnostic performance of LLGP-EITB for porcine $T$. solium cysticercosis was evaluated in Peruvian pigs, and the cross-reaction of the assay to $T$. hydatigena was not known [13]. An individual serum sample was considered positive for $T$. solium antibodies if it was positive to both $\mathrm{rT} 24 \mathrm{H}$ and native LLGP antigens.

\section{Statistical analysis}

Risk factors for porcine T. solium cysticercosis in the communities of Dak Lak Province were identified using logistic regression. In this study, the outcome of interest was a dichotomous variable where households where at least one pig was $T$. solium cysticercosis-positive were assigned a value of 1 , and 0 otherwise. The association between each of a set of household-level candidate explanatory variables from the questionnaires and the outcome of interest were tested using unconditional odds 
ratios and the chi-square test. All explanatory variables associated with the outcome of being $T$. solium cysticercosis positive at an alpha level of $<0.20$ using the chi-square test were selected for inclusion in the multivariable model.

A frequentist fixed-effects logistic regression model was developed in which the probability of a household having at least one cysticercosis-positive pig was parameterized as a function of the explanatory variables with significance of the chi-square test at $P<0.20$, as described above. The significance of each explanatory variable in the model was tested using the chi-square test. Explanatory variables that were not statistically significant were removed from the model one at a time, beginning with the least significant, until the estimated regression coefficients for all the explanatory variables retained in the model were significant at an alpha level of $<0.05$.

To account for the hierarchical structure of the data (households within villages) a village-level random effect term $\left(V_{i}\right)$, was included in the model as shown in Equation 1. District was not a significant predictor of household level T. solium cysticercosis status at the alpha level of 0.05 and was therefore not considered further in the mixed-effects model.

$$
\begin{aligned}
\log \left[\frac{p i}{1-p i}\right]= & \beta_{0}+\beta_{1} x_{1 i}+\cdots \\
& +\beta_{m} x_{m i}+V_{i}+\varepsilon_{i}
\end{aligned}
$$

Due to the low prevalence of $T$. solium cysticercosis (12 of 1281 pigs were positive) regression coefficients for the mixed-effects logistic regression model were estimated using a Bayesian approach implemented in JAGS $[18,19]$. Flat (uninformed) prior distributions were assumed for the intercept $\beta_{0}$ and each of the regression coefficients for the fixed effects $\beta_{1} \cdots \beta_{m}$. The village-level random effect term $\left(V_{i}\right)$ was parameterized as having a normal distribution with mean 0 and precision (inverse variance) $\tau$.

For each of the Bayesian regression analyses we ran the Markov chain Monte Carlo sampler for 40,000 iterations and discarded the first 1000 'burn-in' samples. Convergence was visually assessed by plotting cumulative path plots for each of the monitored parameters [20, 21] and quantified using the Raftery \& Lewis convergence diagnostic $[22,23]$. Parallel chains were run using diverse initial values to ensure that convergence was achieved to the same distribution [24]. Posterior sample sizes were determined by running sufficient iterations to ensure that the Monte Carlo standard error of the mean was at least one order of magnitude smaller than the posterior standard deviation for each parameter of interest.
The results of the final mixed-effects logistic regression model are reported in terms of adjusted odds ratios for each explanatory variable. Assuming a causal relationship between a given explanatory variable and porcine cysticercosis, an adjusted odds ratio [and its 95\% credible interval $(\mathrm{CrI})]$ of $>1$ indicates that, after adjusting for other variables in the model, the explanatory variable increased the risk of a pig being cysticercosis positive. An adjusted odds ratio (and its 95\% CrI) of $<1$ indicates that exposure to the explanatory variable was protective, and an OR of 1 indicates that the variable was not associated with porcine cysticercosis risk.

Statistical analyses were performed using the packages R2jags [25] and coda [26] implemented in $\mathrm{R}$ version 3.3.0 [27].

\section{Results}

\section{General description of study population}

A total of 1324 pig serum samples were collected in Krong Nang, M'Drak and Buon Don districts in Dak Lak Province. Of these, 1281 samples were eligible for further examination as 43 samples were excluded from analysis due to hemolysis or missing questionnaire information. All 1281 serum samples were screened in pools of 4 using the rT24H-EITB assay, and 10 pool samples were identified as positive. Twelve single samples among the 10 positive pool samples were positive for $T$. solium antibodies using the rT24H-EITB assay and all 12 single samples were positive using LLGP-EITB. The prevalence of $T$. solium cysticercosis in pigs in the study districts was 0.94 (95\% CI: 0.51-1.68) per 100 pigs at risk. The 12 positive pigs belonged to 11 households in the three study districts. Of 203 households visited in M'Drak district, 9 (4.43\%, 95\% CI: 2.17-0.85\%) possessed T. solium seropositive pigs. Among 70 visited households in Krong Nang district, two (2.8\%, 95\% CI: $0.49-10.8 \%)$ possessed T. solium cysticercosis positive pigs, and no seropositive pigs were identified in 135 households in Buon Don District.

The hierarchical structure of the data in this study is shown in Table 2. The 1281 pigs were from 408 households and, within each household, an average of three pigs were sampled (minimum 1; maximum 24).

Table 2 Structure of the data from 1281 study pigs from six villages in M'Drak, Buon Don and Krong Nang districts

\begin{tabular}{llll}
\hline Level & Number & \multicolumn{2}{l}{ Number at the next highest level } \\
\cline { 3 - 4 } & & Mean & Range \\
\hline Districts & 3 & - & - \\
Villages & 6 & 2 & 2 \\
Households & 408 & 68 & $21-135$ \\
Pigs $^{a}$ & 1281 & 3 & $1-24$ \\
\hline
\end{tabular}

${ }^{a} A$ total of 1281 pigs recruited in this study. The mean no. of pigs per household was three (range 1-24) 
Of the 408 households, 266 (65\%, 95\% CI: 60-70\%) used a pit latrine; however, most of these latrines were of temporary construction, which animals were able to access. A total of 35\% (95\% CI: 30-40\%) of householders responded that their family members practiced outdoor defection; children typically defaecated around the main household building while adults defaecated some distance from the main household building, within the confines of the household property. Seventy-six percent (95\% CI: $72-80 \%$ ) of households had a pigsty and $58 \%$ (95\% CI: 53-63\%) confined their pigs at all times. Free-roaming pigs habitually ranged around the village to seek food and to return to their litters located under stilt housing in the afternoon. Approximately 7\% (95\% CI: $5-10 \%)$ of households used water sourced from lakes, streams or ponds for their pigs. The remaining households used either rainwater or water from wells or pipes. Dogs were kept in $63 \%$ (95\% CI: 59-68\%) of the households that owned pigs (Table 3 ).

Among the 1281 pigs that were sampled, $41 \%$ (95\% CI: $38-44 \%$ ) were of local breed (Soc). A total of $27 \%$ (95\% CI: 24-29\%) of the sampled pigs were reported to regularly consume human faeces. A little over half of the pigs were routinely offered raw, unwashed vegetables (57\%; 95\% CI: 54-59\%). Commercial and/or homemade bran was offered to $89 \%$ (95\% CI: $86-91 \%$ ) of pigs. The small proportion of pigs that were not supplied bran (11\%, 95\% CI: 9-12\%) were scavenging for the most part (Table 4).

\section{Risk factors for porcine $T$. solium cysticercosis}

Of the data recorded using the questionnaire, we identified two factors associated with a pig's likelihood of being T. solium cysticercosis positive: (i) frequent coprophagy of human faeces; and (ii) scavenging for food. Estimated regression coefficients for the mixed-effects logistic regression model provided in Table 5. After adjusting for the other explanatory variables in the model, the odds of a household where pigs routinely consumed human faeces being T. solium cysticercosis positive was 2.57 (95\% CrI: 1.22-4.66) times that of a household where pigs did not consume human faeces. The odds ratio for a household where pigs routinely scavenged for food was 1.98 (95\% CrI: 0.55-4.74).

\section{Discussion}

In low-income rural communities of Vietnam, a substantial proportion of the human population practice open defaecation and uncooked pork and beef consumption is relatively common. Allowing pigs to roam freely is a common husbandry practice in the region $[4,5,9,28,29]$. Despite all relevant risk factors for cysticercosis being present in each of the communities in this study, the prevalence of $T$. solium cysticercosis was low at
Table 3 General description of household data

\begin{tabular}{|c|c|c|}
\hline Characteristic & Frequency & Percentage $(95 \% \mathrm{Cl})$ \\
\hline Number of households & 408 & - \\
\hline \multicolumn{3}{|l|}{ District } \\
\hline M'Drak & 203 & $50(45-55)$ \\
\hline Buon Don & 135 & $33(28-38)$ \\
\hline Krong Nang & 70 & $17(14-21)$ \\
\hline \multicolumn{3}{|l|}{ Presence of pit latrine } \\
\hline Yes & 266 & $65(60-70)$ \\
\hline No & 142 & $35(30-40)$ \\
\hline \multicolumn{3}{|l|}{ Presence of pigsty } \\
\hline Yes & 311 & $76(72-80)$ \\
\hline No & 97 & $24(20-28)$ \\
\hline \multicolumn{3}{|c|}{ Are pigs permitted to roam freely? } \\
\hline Yes & 172 & $42(37-47)$ \\
\hline No & 236 & $58(53-63)$ \\
\hline \multicolumn{3}{|l|}{ Source of water for pigs } \\
\hline Pipe/well/rain water & 379 & $93(90-95)$ \\
\hline Lake/stream/pond & 29 & $7.0(4.9-10)$ \\
\hline \multicolumn{3}{|l|}{ Owning dogs } \\
\hline Yes & 259 & $63(59-68)$ \\
\hline No & 149 & $37(32-41)$ \\
\hline \multicolumn{3}{|c|}{ Household observing proglottids in dog faeces } \\
\hline Yes & 113 & $44(37-50)$ \\
\hline No & 146 & $56(50-62)$ \\
\hline \multicolumn{3}{|l|}{ Faeces of dogs treated } \\
\hline Yes & 3 & $1.1(0.3-4.6)$ \\
\hline No & 256 & 99 (96-99) \\
\hline \multicolumn{3}{|l|}{ Site of dog defecation } \\
\hline Inside compound & 192 & $74(68-79)$ \\
\hline Outside compound & 67 & $26(21-32)$ \\
\hline
\end{tabular}

0.94 (95\% CI: 0.51-1.68) cases per 100 pigs at risk. A similar, low prevalence of cysticercosis has been observed not only in Dak Lak Province but in other regions of Vietnam [4, 6, 30]. In 1994 Huan, in a cross-sectional study of pigs submitted for slaughter from 12 provinces in the south of Vietnam, reported a prevalence of 0.90 (95\% CI: 0.45-1.76) cases per 100 pigs at risk [6]. In three studies carried out in 10 provinces in the north of Vietnam between 1999 and 2003, the prevalence of cysticercosis ranged from 0 to 0.06 cases per 100 pigs at risk [31-33]. In the Province of Bac Ninh in the north of Vietnam, a known foci of T. solium in humans, carcass examination of 26 village pigs identified no cases of $T$. solium cysticercosis. Instead, 10 pigs were positive for T. hydatigena cysticerci [34]. These findings are in agreement with those of Conlan et al. [35], who reported a low 
Table 4 General description of pig data

\begin{tabular}{|c|c|c|}
\hline Characteristic & Frequency & Percentage $(95 \% \mathrm{Cl})$ \\
\hline Number of pigs & 1281 & - \\
\hline \multicolumn{3}{|l|}{ Sex } \\
\hline Male & 536 & $42(39-45)$ \\
\hline Female & 745 & $58(55-61)$ \\
\hline \multicolumn{3}{|l|}{ Age (months) } \\
\hline$<4$ & 497 & $39(36-42)$ \\
\hline $4-12$ & 665 & $52(49-55)$ \\
\hline$>12$ & 119 & $9.3(7.8-11)$ \\
\hline \multicolumn{3}{|l|}{ Breed } \\
\hline Imported breed & 522 & $41(38-44)$ \\
\hline Local breed (Soc) & 759 & $59(56-62)$ \\
\hline \multicolumn{3}{|l|}{ Allowed to roam freely } \\
\hline Yes & 406 & $32(29-34)$ \\
\hline No & 875 & $68(66-71)$ \\
\hline \multicolumn{3}{|l|}{ Human coprophagy } \\
\hline Yes & 340 & $27(24-29)$ \\
\hline No & 941 & $73(71-76)$ \\
\hline \multicolumn{3}{|l|}{ Main food } \\
\hline Commercial/handmade bran & 1145 & $89(86-91)$ \\
\hline Scavenging & 136 & $11(9.0-12)$ \\
\hline \multicolumn{3}{|c|}{ Provision of raw, unwashed vegetables ${ }^{a}$} \\
\hline Yes & 723 & $57(54-59)$ \\
\hline No & 555 & $43(41-46)$ \\
\hline
\end{tabular}

${ }^{a}$ Three cases was excluded from analysis

prevalence of $T$. solium cysticercosis of $0.8 \%$ in village pigs in Laos with a relatively high prevalence of $T$. hydatigena (22 cases per 100 pigs at risk) and a high prevalence of T. solium taeniasis. Conlan et al. [35] hypothesized that $T$. hydatigena is likely to cross-protect pigs from $T$. solium infection.
In Vietnam the prevalence of T. hydatigena cysticercosis in pigs has been reported to be high, ranging between $25-38 \%$ in the north and the prevalence has been strongly correlated with the presence of $T$. hydatigena infection in dogs [36]. In addition, most of the households that owned pigs in the three study districts also kept dogs and approximately one half of the households owning dogs reported that they had observed proglottids in their dog's faeces (Table 3). All of the 10 free-roaming village pigs that were backyard slaughtered had $T$. hydatigena cysticerci present in the mesentery, stomach, spleen, and liver (personal observation from fieldwork). It is our inference that the relatively high prevalence of $T$. hydatigena infection in dogs is likely to be a major source of $T$. hydatigena cysticercosis in pigs. If this is true a cautioned approach to cysticercosis control in pigs would be advised if $T$. hydatigena were to be targeted through pig confinement and canine deworming programs. Eliminating or reducing pig exposure to $T$. hydatigena would likely result in an increase in the observed prevalence of $T$. solium in non-compliant free-roaming pigs, providing an increased public health risk to the community.

Among the investigated households that owned pigs, a little under one-quarter did not have a pigsty and most of the pigs that were kept were of the local breed (Table 4). Local breeds are preferred due to their ability to thrive under harsh raising conditions and poor feeding [12]. Allowing pigs to free-roam for food was common practice (Table 3). The odds of a household where pigs routinely scavenged for food being seropositive for T. solium cysticercosis was 1.98 (95\% CrI: 0.55-4.74) times greater than the odds of a household where pigs were fed commercial and/or homemade bran (Table 5). Similarly, the odds ratio for a household where pigs routinely consumed human faeces being seropositive was 2.57 (95\% CrI: 1.22-4.66) times greater than the odds of a household where this

Table 5 Risk factors associated with T. solium cysticercosis positive in pigs

\begin{tabular}{|c|c|c|c|c|c|}
\hline \multirow{2}{*}{$\begin{array}{l}\text { Explanatory } \\
\text { variable }\end{array}$} & \multicolumn{2}{|c|}{ Number of pigs } & \multirow{2}{*}{$\begin{array}{l}\text { Regression } \\
\text { coefficient (SD) }\end{array}$} & \multirow{2}{*}{$\begin{array}{l}\mathrm{MC} \\
\text { error }\end{array}$} & \multirow{2}{*}{$\begin{array}{l}\text { Adjusted OR } \\
(95 \% \text { Crl) }\end{array}$} \\
\hline & Positive & Total & & & \\
\hline Intercept & 12 & 1281 & $-5.9800(1.5480)$ & 0.006 & - \\
\hline \multicolumn{6}{|c|}{ Coprophagy of human faeces: } \\
\hline No & 3 & 941 & Reference & & \\
\hline Yes & 9 & 340 & $2.6600(0.8470)$ & 0.004 & $2.57(1.22-4.66)$ \\
\hline \multicolumn{6}{|l|}{ Kind of food } \\
\hline Bran & 6 & 1145 & Reference & & \\
\hline Scavenging & 6 & 136 & $2.1400(1.0320)$ & 0.003 & $1.98(0.55-4.74)^{2}$ \\
\hline Random effects ${ }^{\mathrm{b}}$ & Estimate & SD & & & \\
\hline Village & 2.18 & 5.77 & & & \\
\hline
\end{tabular}

Interpretation: The odds of T. solium cysticercosis positive for pigs that scavenged food was 1.98 (95\% credible interval $0.55-4.74)$ times that of pigs that did not scavenge for food

${ }^{\mathrm{b}}$ Variance and standard deviation (SD) of the variance of the village-level random effect 
practice was not habitual. It is known that risk factors for transmission and circulation of porcine cysticercosis are numerous and may vary in different settings. We found that allowing pigs to free-roam and allowing pigs to routinely consume human faeces were associated with $T$. solium exposure, consistent with other studies [37-39]. Research on the epidemiological characteristics of porcine cysticercosis in Peru [40], Mozambique [41] and Mexico [42] found that older pigs were more likely to show evidence of exposure to T. solium compared to younger pigs, an association not identified in this study. Similarly, while studies from Zambia [43], Mexico [44], and Tanzania [45] showed that the prevalence of T. solium exposure was higher in male pigs compared with females, this association was not identified in this study. In this study, both of the risk factors identified are modifiable, which means that there exists an opportunity to decrease the prevalence of porcine cysticercosis even further. We propose that a combination of intervention measures including education and public awareness campaigns, strategies to reduce coprophagy among pigs and enhanced meat inspection (particularly backyard slaughtered stock) are likely to have the greatest impact on porcine cysticercosis risk with positive secondary effects on human health [46]. For this to be successful there is a need for commitment and support from local and/or central veterinary and medical health authorities.

\section{Conclusions}

The prevalence of porcine T. solium cysticercosis in this study was low at 0.94 (95\% CI: $0.51-1.68)$ cases per 100 pigs at risk, in agreement with other studies conducted throughout Vietnam. Scavenging of food and coprophagy were associated with $T$. solium cysticercosis risk. Both of these characteristics are modifiable providing the opportunity to decrease the prevalence of porcine cysticercosis even further.

\section{Abbreviations}

EITB: enzyme-linked immunoelectrotransfer blot; LLGP: lentil lectin purified glycoprotein; rT24H: recombinant antigen $\mathrm{T} 24 \mathrm{H}$

\section{Acknowledgements}

We are grateful to the Institute of Biotechnology and Environment Tay Nguyen University for providing resources and facilities for the fieldwork. We thank to local veterinarian staff at M'Drak, Krong Nang and Buon Don district for assisting in sample collection. The authors are most thankful to Ms. Nguyen Thi Ngoc Hien, Ms. Long Khanh Linh, and Ms. Nguyen Thi Lan Huong, who assisted with laboratory work.

\section{Funding}

This research was self-funded by RJT. This work was done with partial support for travel from the Faculty of Veterinary and Agricultural Sciences, University of Melbourne, Australia. DNN received PhD scholarship from Australia Awards Scholarships, Department of Foreign Affairs and Trade, Australia Government. The materials for serological diagnosis were provided by the Division of Parasitic Diseases and Malaria, Centers for Disease Control and Prevention, Atlanta, Georgia, United States of America.
Availability of data and materials

All relevant data are included within this published article.

\section{Authors' contributions}

DNN designed study, analyzed data and wrote manuscript; JN: provided material, designed study and edited paper; KB: assisted laboratory work, MAS: assisted with analyses of data and edited paper; SH: provided material, designed study and edited paper; RJT provided material, supervised study and edited paper. All authors read and approved the final manuscript.

\section{Ethics approval and consent to participate}

This study was reviewed and approved by the Animal Ethics and scientific committee, Tay Nguyen University (reference number 50.KCNTY), and conducted under the supervision of the local center for animal health, Dak Lak, Vietnam. Verbal consent was obtained to participate in the study. The findings and conclusions in this report are those of the author(s) and do not necessarily represent the official position of the Centers for Disease Control and Prevention.

\section{Consent for publication}

Not applicable.

\section{Competing interests}

The authors declare that they have no competing interests.

\section{Publisher's Note}

Springer Nature remains neutral with regard to jurisdictional claims in published maps and institutional affiliations.

\section{Author details}

${ }^{1}$ Faculty of Veterinary and Agricultural Sciences, University of Melbourne, Parkville, Victoria 3052, Australia. ${ }^{2}$ Faculty of Animal Sciences and Veterinary Medicine, Tay Nguyen University, Buon Ma Thuot, Dak Lak, Vietnam. ${ }^{3}$ Division of Parasitic Diseases and Malaria, Centers for Disease Control and Prevention, Atlanta, Georgia, USA. ${ }^{4}$ Department of Livestock, Montana Veterinary Diagnostic Lab, Bozeman, Montana, USA.

Received: 3 April 2018 Accepted: 7 June 2018

Published online: 22 June 2018

\section{References}

1. FAO/WHO. Multicriterial-based Ranking for Risk Management of Food-borne Parasites. Microbiological Risk Assessment Series No 23. In: Rome: WHO Press; 2014.

2. Donadeu M, Lightowlers MW, Fahrion AS, Kessels J, Donadeu M, Lightowlers MW, et al. Taenia solium: WHO endemicity map update. Wkly Epidemiol Rec. 2016;49/50:595-9.

3. Aung AK, Spelman DW. Taenia solium taeniasis and cysticercosis in southeast Asia. Am J Trop Med Hyg. 2016;94:947-54.

4. Ng-Nguyen D, Stevenson MA, Traub RJ. A systematic review of taeniasis, cysticercosis and trichinellosis in Vietnam. Parasit Vectors. 2017;10:150.

5. Huong NT. Taeniasis and cysticercosis in a selected group of inhabitants from a mountainous province in North Vietnam. Antwerpen, Belgium: Prince Leopold Institute of Tropical Medicine; 2006.

6. Huan L Van. Parasitic helminths in pigs in several southern provinces and preventative measures National Institution of Veterinary Research; 1994. http:// luanan.nlv.gov.vn/luanan?a=d\&d=TTkFvmnEdmia1994.1.2\&e=-\%2D-\%2D-\%2Dvi-20\%2D-1\%2D-img-txlN-\%2D-\%2D-\%2D-\#. Accessed 10 Nov 2015.

7. Ng-Nguyen D, Stevenson MA, Dorny P, Gabriël S, Van VT, Nguyen VT, et al. Comparison of a new multiplex real-time PCR with the Kato-Katz thick smear and copro-antigen ELISA for the detection and differentiation of Taenia spp. in human stools. PLoS Negl Trop Dis. 2017;11:e0005743.

8. Nguyen TH. Evaluation of market opportunities for producing local pigs in Dak Lak. Tay Nguyen J Sci. 2009:5:21-6.

9. Van De N, Le TH, Lien PTH, Eom KS. Current status of taeniasis and cysticercosis in Vietnam. Korean J Parasitol. 2014:52:125-9.

10. Asaava LL, Kitala PM, Gathura PB, Nanyingi MO, Muchemi G, Schelling E. A survey of bovine cysticercosis/human taeniosis in Northern Turkana District, Kenya. Prev Vet Med. 2009;89:197-204. 
11. Noh J, Rodriguez S, Lee YM, Handali S, Gonzalez AE, Gilman RH, et al. Recombinant protein- and synthetic peptide-based immunoblot test for diagnosis of neurocysticercosis. J Clin Microbiol. 2014;52:1429-34.

12. Tsang VCW, Brand JA, Boyer AE. An enzyme-linked immunoelectrotransfer blot assay and glycoprotein antigens for diagnosing human cysticercosis (Taenia solium). J Infect Dis. 1989:159:50-9.

13. Gonzalez AE, Cama V, Gilman RH, Tsang VCW, Pilcher JB, Chavera A, et al. Prevalence and comparison of serologic assays, necropsy, and tongue examination for the diagnosis of porcine cysticercosis in Peru. Am J Trop Med Hyg. 1990;43:194-9.

14. Hancock K, Pattabhi S, Whitfield FW, Yushak ML, Lane WS, Garcia HH, et al. Characterization and cloning of T24, a Taenia solium antigen diagnostic for cysticercosis. Mol Biochem Parasitol. 2006;147:109-17.

15. Handali S, Klarman M, Gaspard AN, Noh J, Lee YM, Rodriguez S, et al. Multiantigen print immunoassay for comparison of diagnostic antigens for Taenia solium cysticercosis and taeniasis. Clin Vaccine Immunol. 2010;17:68-72.

16. Rodriguez S, Wilkins P, Dorny P. Immunological and molecular diagnosis of cysticercosis. Pathog Glob Health. 2012;106:286-98.

17. Jayashi CM, Gonzalez AE, Castillo Neyra R, Rodríguez S, García HH, Lightowlers MW. Validity of the enzyme-linked immunoelectrotransfer blot (EITB) for naturally acquired porcine cysticercosis. Vet Parasitol. 2014;199:42-9.

18. Kruschke JK. Review of doing Bayesian data analysis: a tutorial with R, JAGS, and Stan (second edition). Clin Neuropsychol. 2017:31:1268-70.

19. Plummer M. JAGS Version 3.4.0 user manual. 2013;0-41. http://www.stats.ox. ac.uk/nicholls/MScMCMC15/jags_user_manual.pdf

20. Yu B, Mykland P. Looking at Markov samplers through cusum path plots: a simple diagnostic idea. Stat Comput. 1998;8:275-86.

21. Robert CP, Casella G. Monte Carlo Statistical Methods. New York, USA: Springer New York; 2004.

22. Raftery AE, Lewis SM. One long run with diagnostics: implementation strategies for Markov Chain Monte Carlo. Stat Sci. 1992;7:493-7.

23. Raftery EA, Lewis MS. How many iterations in the Gibbs Sampler? In: Benardo J, Berger J, Dawid A, Smith A, editors. Bayesian Stat 4. New York: The Clarendon Press and: Oxford University Press; 1992. p. 763-74

24. Gelman A. Inference and monitoring convergence. In: Gilks W, Richardson S, Spiegelhalter D, editors. Markov Chain Monte Carlo Pract. London: Chapman \& Hall; 1996. p. 131-43.

25. Su Y-S, Yajima M. Using R to Run "JAGS." 2015. https://cran.r-project.org/ web/packages/R2jags/R2jags.pdf

26. Plummer M, Best N, Cowles K, Vines K, Sarkar D, Bates D, et al. Coda: Output Analysis and Diagnostics for MCMC. R News. 2016;6:7-11.

27. Team. R Core. In: R: A Language and Environment for Statistical Computing [Internet]. Vienna, Austria: R Foundation for Statistical Computing; 2017. http://www.r-project.org.

28. Anh Tuan P, Dung TTK, Nhi VA. Sero-epidemiological investigation of cysticercosis in the southern provinces. J Malar Parasite Dis Control. 2001:4:81-7

29. Trung DD, Praet N, Cam TDT, Lam BVT, Manh HN, Gabriël S, et al. Assessing the burden of human cysticercosis in Vietnam. Trop Med Int Health. 2013; 18:352-6.

30. Willingham AL, Van De N, Doanh NQ, Cong D, Dung TV, Dorny P, et al. Current status of cysticercosis in Vietnam. Southeast Asian J Trop Med Public Health. 2003;34:35-50.

31. Van KP, Luc P. Veterinary Parasitology. Hanoi: Hanoi Agricultural Publishing House; 1996.

32. Doanh NQ, Holland W, Vercruysse J, Van DN. Results of survey on cysticercosis pig in some northern provinces in Vietnam. J Malar Parasite Dis Control. 2002;6:76-82.

33. De N Van, Chau L Van, Son DT, Chuyen LT, Hop NT, Vien HV, et al. Taenia solium survey in Hanoi. J Malar Parasite Dis Control. 2004;6:93-99.

34. Doanh NQ, Kim NT, De NV, Lung NL. Result of survey on taeniasis and cysticercosis humans and pigs in Bac Ninh and Bac Kan provinces. Vet Sci Tech. 2002:9:46-9.

35. Conlan JV, Vongxay K, Khamlome B, Dorny P, Sripa B, Elliot A, et al. A crosssectional study of Taenia solium in a multiple taeniid-endemic region reveals competition may be protective. Am J Trop Med Hyg. 2012;87:281-91.

36. Lan NTK, Quyen NT, Hoat PC. The correlation between the prevalence of the tapeworm Taenia hydatigena in dogs and their larvae Cysticercus tenuicollis in cattle and pigs - the effect of tapeworm treatment in dogs. Vet Sci Tech. 2011:18:60-5.
37. Ngowi HA, Kassuku AA, Maeda GEM, Boa ME, Carabin H, Willingham AL. Risk factors for the prevalence of porcine cysticercosis in Mbulu District, Tanzania. Vet Parasitol. 2004;120:275-83.

38. Sikasunge CS, Phiri IK, Phiri AM, Dorny P, Siziya S, Willingham AL. Risk factors associated with porcine cysticercosis in selected districts of Eastern and Southern provinces of Zambia. Vet Parasitol. 2007;143:59-66.

39. Pouedet MSR, Zoli AP, Nguekam VL, Assana E, Speybroeck N, et al. Epidemiological survey of swine cysticercosis in two rural communities of West-Cameroon. Vet Parasitol. 2002;106:45-54.

40. Lescano AG, García HH, Gilman RH, Guezala MC, Tsang VCW, Gavidia CM, et al. Swine cysticercosis hotspots surrounding Taenia solium tapeworm carriers. Am J Trop Med Hyg. 2007;76:376-83.

41. Pondja A, Neves L, Mlangwa J, Afonso S, Fafetine J, Willingham AL 3rd, et al. Prevalence and risk factors of porcine cysticercosis in Angonia District, Mozambique. PLoS Negl Trop Dis. 2010;4:e594.

42. Sarti Gutierrez E, Schantz PM, Aguilera J, Lopez A. Epidemiologic observations on porcine cysticercosis in a rural community of Michoacan State, Mexico. Vet Parasitol. 1992;41:195-201.

43. Sikasunge CS. The prevalence and transmission risk factors of porcine cysticercosis in eastern and southern provinces of Zambia. MSc Thesis: University of Zambia, Lusaka, Zambia; 2005.

44. García HH, Gilman RH, Gonzalez AE, Verastegui M, Rodriguez S, Gavidia C, et al. Hyperendemic human and porcine Taenia solium infection in Perú. Am J Trop Med Hyg. 2003;68:268-75.

45. Shonyela SM, Mkupasi EM, Sikalizyo SC, Kabemba EM, Ngowi HA, Phiri I. An epidemiological survey of porcine cysticercosis in Nyasa District, Ruvuma Region, Tanzania. Parasite Epidemiol Control. 2017;2:35-41.

46. Gabriël S, Dorny P, Mwape KE, Trevisan C, Braae UC, Magnussen P, et al. Control of Taenia solium taeniasis/cysticercosis: The best way forward for sub-Saharan Africa? Acta Trop. 2017;165:252-60.

\section{Ready to submit your research? Choose BMC and benefit from:}

- fast, convenient online submission

- thorough peer review by experienced researchers in your field

- rapid publication on acceptance

- support for research data, including large and complex data types

- gold Open Access which fosters wider collaboration and increased citations

- maximum visibility for your research: over $100 \mathrm{M}$ website views per year

At BMC, research is always in progress.

Learn more biomedcentral.com/submissions 